\title{
Gender Differences are Noted in Factors Associated with Insomnia in Older Adult Outpatients: A Cross-Sectional Study
}

\author{
Yu-Ting Peng \\ Kaohsiung Veterans General Hospital \\ Ying-Hsin Hsu \\ Kaohsiung Veterans General Hospital \\ Ming-Yueh Chou \\ Kaohsiung Veterans General Hospital \\ Che-sheng Chu \\ Kaohsiung Veterans General Hospital \\ Chen-San Su \\ Kaohsiung Veterans General Hospital \\ Chih-Kuang Liang ( $\nabla$ ck.vghks@gmail.com ) \\ Kaohsiung Veterans General Hospital

\section{Yu-Chun Wang} \\ Kaohsiung Veterans General Hospital

\section{Tsan Yang} \\ Meiho University \\ Liang-Kung Chen \\ National Yang Ming Chiao Tung University

\section{Yu-Te Lin} \\ Kaohsiung Veterans General Hospital
}

\section{Research Article}

Keywords: Associated factors, gender differences, insomnia, older adults

Posted Date: July 26th, 2021

DOI: https://doi.org/10.21203/rs.3.rs-677626/v1

License: (c) (i) This work is licensed under a Creative Commons Attribution 4.0 International License. Read Full License 


\section{Abstract}

Background: Insomnia is a common sleep disturbance in older adults and is associated with many poor health outcomes. This study aimed to investigate the relevance of insomnia in older adult outpatients and to analyze differences between genders in factors associated with insomnia.

Methods: This cross-sectional study was conducted in the outpatient clinics of a tertiary hospital in Southern Taiwan from July to September 2018. A total of 400 consecutive subjects aged 60 years or older were recruited. Insomnia was defined as a score of $>6$ points on the Athens Insomnia Scale (AIS). Socio-demographics, health behaviors and clinical data were collected by face-to-face interview. Multivariable logistic regression was adopted for statistical analysis of the entire sample and stratified by gender.

Results: Participants' mean age was $74.74 \pm 8.54$ years, and the majority (93\%) had more than one chronic disease. The prevalence of insomnia accounted for $30 \%$ (120/400) of all subjects, with males $22.9 \%(46 / 201)$ and females $37.2 \%$ (74/199). Gender, appetite, exercise, depressive symptoms, and sleep-related conditions such as short sleep duration, sleeping pills usage, medium-high risk of obstructive sleep apnea(OSA) and restless leg syndrome(RLS) were factors associated with insomnia in older adults. Exercise, sleeping pills usage, and RLS had an independent association with insomnia only in men, while appetite was associated with insomnia in women only.

Conclusions: Insomnia is highly prevalent among older adults, predominantly females. Significant differences are found between genders in factors associated with insomnia. Understanding gender differences may help clinicians to modify associated factors when managing older adults with insomnia.

\section{Background}

Since the world is facing an aging society, much attention has been paid to sleep problems in older adults. Insomnia is a common sleep-related complaint among older adults, whether it is reported as subjective symptoms or defined as a sleep disorder according to recognized diagnostic systems. Although the definition varies by diagnostic criteria, about $30-48 \%$ of older adults are estimated to have insomnia symptoms, and the prevalence of insomnia disorder is around $12-20 \%$ [1]. Insomnia is not only associated with poor health outcomes such as cardiovascular disease [2], cognitive impairment [3], and mental disorders [4], but also reduces the quality of life [5] and poses a significant socioeconomic burden on society. Thus, finding potentially modifiable factors associated with insomnia is the first step in preventing insomnia-related consequences.

Evidence has revealed numerous factors associated with insomnia, including demographic, psychological and social characteristics. Women older than 45 years [6, 7], older adults who are divorced, separated or widowed [6], and those with lower levels of education and/or income, smoking, alcohol use, and reduced physical activity[6, 8] are factors associated with higher rates of insomnia in older adults. Clinical conditions such as physical illness [9], depression [10, 11], and some primary sleep disorders like obstructive sleep apnea (OSA) and restless leg syndrome (RLS) are also associated with or are comorbid with insomnia in various populations and clinical settings $[1,12,13]$. Nevertheless, the effects of insomnia on lifestyle and sleep behavior in older adults are not well established. Among the factors, female gender is frequently reported as a significant factor associated with insomnia[9, 10, 14-17]. However, few studies have investigated gender differences in specific factors associated with insomnia $[18,19]$, especially not in older adults. Little is known about the influence of gender in factors associated with insomnia in older adults.

This study aimed to investigate factors associated with insomnia in older adult outpatients and also stratified by gender to evaluate the influence of gender on the insomnia-associated factors. 


\section{Methods}

\section{Study design and participants}

This is a cross-sectional prospective study conducted at the Geriatric, Neurology, and Psychiatry outpatient clinics of Kaohsiung Veterans General Hospital (a tertiary medical center), Taiwan, from July to September 2018. Older adults with severe cognitive impairment (Clinical Dementia Rating 3 or above), psychiatric disorders, or challenging communication were excluded. A total of 400 older adult subjects, aged 60-97 years, were recruited. Face-to-face interviews were conducted by well-trained nursing researchers using structured questionnaires, including demographic data, personal lifestyle habits, physical and mood function, and sleep-related characteristics.

\section{Ethical considerations}

According to the principles of the Declaration of Helsinki, the study protocol was reviewed and approved by the internal review board of Kaohsiung Veterans Hospital, Taiwan (approval number VGHKS18-CT6-15). Participants were volunteers and all provided signed informed consent at the study inception. Participants also had the right to withdraw from participation at any time.

\section{Data collection/Main measures}

\section{Demographic, lifestyle and clinical data}

The study questionnaire for socio-demographic data included age, sex, educational level, marital and residential status, and occupational activity. Simple dichotomous (yes-no) questions were used without further quantification for lifestyle factors, including current smoking status or alcohol drinking, coffee drinking, poor appetite within recent 3 months, exercise habits, and afternoon naps. Among clinical data, obesity was defined as body mass index (BMI) $\geq 27$ $\mathrm{kg} / \mathrm{m} 2$ according to criteria listed by the Department of Health in Taiwan [20], since that Asians seem to bear significant risks at lower BMI cut-off than Caucasians [21, 22]. Data on chronic diseases and the diagnosis of dementia were collected from patients' medical charts and self-reported by patients and their families. Depressive symptoms and physical function were screened using the Geriatric Depression Scale-5 (GDS-5) [23, 24] and activities of daily living (by Katz Index) [25].

\section{Definition of insomnia}

Insomnia was defined by the Athens Insomnia Scale (AIS), a self-reported questionnaire designed to evaluate sleep difficulty. It consists of eight items, including sleeping time, sleep disruptions, premature awakening, total sleeping time, overall sleep quality, daytime physical and mental function, and daytime sleepiness. Each item is rated on a scale of 0-3 points ( 0 for no problems and 3 for severe insomnia-related symptoms). A cutoff value of $>6$ points is used to define insomnia. The high reliability and accuracy of the AIS have been reported in previous validation studies [26], and the Chinese version validated in 2009 also showed high internal consistency [27].

\section{Covariates for sleep-related problems}


Sleep-related problems, including sleep duration, sleeping pills usage, obstructive sleep apnea (OSA), and restless leg syndrome (RLS), were screened through yes/no questions or structured questionnaires. Self-reported actual duration of sleep (hours per night) within the last one month excluding the time in bed without sleep was recorded. The sleep duration was then grouped into long and normal sleep duration ( $>6$ hours) and short sleep duration $(<6$ hours) [28]. STOP-Bang Sleep Apnea Questionnaire was used to screen for OSA. Scores of 0-2, 3-4, and 5-8 are categorized as low, moderate, and high risk for OSA $[29,30]$. A four-question set recommended by the International Restless Legs Syndrome Study Group (IRLSSG) was used to define RLS [31]. Subjects who met all four criteria were classified into the RLS group.

\section{Statistical analysis}

Socio-demographic and clinical data are presented as means +/- SD or frequencies and percentages. Comparisons were made between insomnia and non-insomnia groups using independent student t-test or one-way analysis of variance (ANOVA) for continuous variables and chi-square test for categorical variables. Factors that demonstrated a significant association $(p<0.05)$ with insomnia in univariable analysis were entered into multivariable logistic regression analysis to identify the independent relationship. Data were further stratified by gender, and analyzed using univariable and multivariable logistic regression analysis. The odds ratio and $95 \%$ confidence interval of each variable was documented. All data were analyzed using the SPSS version 22 (IBM, Armonk, NY, USA). Two-tailed p-values of $<0.05$ were considered to be statistically significant.

\section{Results}

\section{Characteristics of study participants with or without insomnia}

Among the 400 subjects who participated in the present study, the mean age was 74.74 years (SD, 8.54), and about one-half (49.8\%) were women (Table 1). A majority (93\%) of participants had more than one chronic disease. The prevalence of insomnia defined by the AIS scale was $30 \%(n=120)$, and approximately $22.9 \%(46 / 201)$ and $37.2 \%$ (74/199) for male and female subjects, respectively. Subjects with insomnia had a higher prevalence of poor appetite and not forming regular exercise habits. Most participants also had short sleep duration, tendency to use sleeping pills, depressive symptoms, and medium-high risk of OSA and RLS.

Table 1. Characteristics of the study participants 


\begin{tabular}{|c|c|c|c|c|}
\hline Characteristics & Total & $\begin{array}{l}\text { Non-Insomnia } \\
(\mathrm{AIS}<6)\end{array}$ & $\begin{array}{l}\text { Insomnia } \\
(A \mid S>=6)\end{array}$ & \\
\hline & $\mathrm{n}=400(\%)$ & $\mathrm{n}=280(\%)$ & $\mathrm{n}=120(\%)$ & $\mathrm{p}$ value \\
\hline Age (years)\# & $74.74 \pm 8.54$ & $74.88 \pm 8.54$ & $74.43 \pm 8.55$ & 0.63 \\
\hline \multicolumn{5}{|l|}{ Sleep duration (hr) } \\
\hline$<=6 \mathrm{hrs}$ & $204(51)$ & $118(57.8)$ & $86(42.2)$ & $<0.001 *$ \\
\hline$>6 \mathrm{hrs}$ & $196(49)$ & $162(82.7)$ & $34(17.3)$ & \\
\hline Gender & & & & $0.002 *$ \\
\hline Male & $201(50.2)$ & $155(77.1)$ & $46(22.9)$ & \\
\hline Female & $199(49.8)$ & $125(62.8)$ & $74(37.2)$ & \\
\hline \multicolumn{5}{|l|}{ Obesity (BMl>=27) } \\
\hline Yes & $98(24.5)$ & $218(72.2)$ & $84(27.8)$ & 0.094 \\
\hline No & $302(75.5)$ & $62(63.3)$ & $36(36.7)$ & \\
\hline Education & & & & 0.061 \\
\hline$<=9$ years (junior) & $242(60.5)$ & $162(66.7)$ & $81(33.3)$ & \\
\hline >= 10 years & 158 (39.5) & $118(75.2)$ & $39(24.8)$ & \\
\hline Marital status & & & & 0.161 \\
\hline Married & $254(63.5)$ & $184(72.7)$ & $69(27.3)$ & \\
\hline Others & $146(36.5)$ & $96(65.3)$ & $51(34.7)$ & \\
\hline Residential status & & & & 0.243 \\
\hline Live alone & $42(10.5)$ & $28(68.3)$ & $13(31.7)$ & \\
\hline \multicolumn{5}{|l|}{ Live with } \\
\hline Live with others & $352(87.8)$ & $249(70.7)$ & $103(29.3)$ & \\
\hline Nursing home & $7(1.8)$ & $3(42.9)$ & $4(57.1)$ & \\
\hline Occupational activity & & & & 0.355 \\
\hline Working & $42(10.5)$ & $32(74.4)$ & $11(25.6)$ & \\
\hline Not working & $358(89.5)$ & $248(69.5)$ & $109(30.5)$ & \\
\hline Current smoking & & & & 0.064 \\
\hline Yes & $32(8.0)$ & $27(84.4)$ & $5(15.6)$ & \\
\hline No & $368(92.0)$ & $253(68.6)$ & $115(31.2)$ & \\
\hline Current alcohol drinking & & & & 0.061 \\
\hline Yes & $8(2.0)$ & $8(100)$ & $0(0)$ & \\
\hline
\end{tabular}




\begin{tabular}{|c|c|c|c|c|}
\hline No & $392(98.0)$ & $272(69.4)$ & $120(30.6)$ & \\
\hline Drink Coffee & & & & 0.678 \\
\hline Yes & $136(34)$ & $97(71.3)$ & $39(28.7)$ & \\
\hline No & $264(66)$ & $183(69.3)$ & $81(30.7)$ & \\
\hline Appetite within 3 months & & & & $<0.001 *$ \\
\hline Fair & $351(87.8)$ & $259(73.8)$ & $92(26.2)$ & \\
\hline Poor & $49(12.2)$ & $21(42.9)$ & $28(57.1)$ & \\
\hline Habitual Exercise & & & & $0.015^{\star}$ \\
\hline Yes (Regular) & $262(65.5)$ & $194(74.0)$ & $68(26.0)$ & \\
\hline No (non or irregular) & $138(34.5)$ & $86(62.3)$ & $52(37.7)$ & \\
\hline Afternoon Nap & & & & 0.707 \\
\hline Yes & $231(57.8)$ & $160(69.3)$ & $71(30.7)$ & \\
\hline No & $169(42.2)$ & $120(71.0)$ & $49(29.0)$ & \\
\hline Sleeping pills usage & & & & $<0.001 *$ \\
\hline Yes & $167(41.2)$ & $98(58.7)$ & $69(41.3)$ & \\
\hline No & $233(58.3)$ & $182(78.1)$ & $51(21.9)$ & \\
\hline Any chronic disease & & & & 0.060 \\
\hline Yes & $372(93.0)$ & $256(68.8)$ & $116(31.2)$ & \\
\hline No & $28(7.0)$ & $24(85.7)$ & $4(14.3)$ & \\
\hline Dementia (documented) & & & & 0.822 \\
\hline Yes & $74(18.5)$ & $51(68.9)$ & $23(31.1)$ & \\
\hline No & $326(81.5)$ & $229(70.2)$ & $97(29.8)$ & \\
\hline Activities of Daily Living & & & & 0.847 \\
\hline Total independence & $365(91.3)$ & $255(69.9)$ & $110(30.1)$ & \\
\hline Dependence & $35(8.2)$ & $25(71.4)$ & $10(28.6)$ & \\
\hline Depression by GDS-5 & & & & $<0.001^{*}$ \\
\hline No $(<2)$ & $304(76.0)$ & $236(77.6)$ & $68(22.4)$ & \\
\hline Yes $(>=2)$ & $96(24.0)$ & $44(45.8)$ & $52(54.2)$ & \\
\hline Obstructive Sleep Apnea (risk) & & & & $0.004^{*}$ \\
\hline Low & $138(34.5)$ & $109(79.0)$ & $29(21.0)$ & \\
\hline Medium-High & $262(65.5)$ & $171(65.3)$ & $91(34.7)$ & \\
\hline Restless Leg Syndrome & & & & $0.001 *$ \\
\hline No & $384(96.0)$ & $275(71.6)$ & $109(28.4)$ & \\
\hline
\end{tabular}


\#Continuous variables are presented as mean \pm SD

* statistical significance with p-value $<0.05$

Abbreviations: BMI - Body Mass Index, GDS-5 - Geriatric Depression Scale-5

\section{Factors associated with insomnia in older adults}

Table 2 shows the factors associated with insomnia determined by univariable and multivariable logistic regression analysis. In univariable analysis, all factors except age were significantly associated with insomnia. In Model 1 of multivariable logistic regression, female gender $(\mathrm{OR}=2.25,95 \% \mathrm{Cl}=1.33-3.86)$, poor appetite within 3 months $(\mathrm{OR}=$ $3.02,95 \% \mathrm{Cl}=1.53-5.95)$, using sleeping pills $(\mathrm{OR}=1.96,95 \% \mathrm{Cl}=1.21-3.19)$, medium-high risk of OSA $(\mathrm{OR}=2.99$, $95 \% \mathrm{Cl}=1.67-5.33)$, depressive symptoms $(\mathrm{OR}=2.69,95 \% \mathrm{Cl}=1.58-4.56)$ and $\mathrm{RLS}(\mathrm{OR}=5.23,95 \% \mathrm{Cl}=1.55-16.40)$ were all independently associated with insomnia, except for non- or irregular exercise. When sleep duration was added as an additional covariate into Model 2, the independent relationship between insomnia and those factors that were significant in Model 1 still existed, including female gender ( $O R=2.42,95 \% \mathrm{Cl}=1.40-4.19)$, poor appetite within 3 months $(\mathrm{OR}=3.59,95 \% \mathrm{Cl}=1.74-7.39)$, sleeping pills usage $(\mathrm{OR}=2.04,95 \% \mathrm{Cl}=1.22-3.40)$, moderate-high risk of OSA (OR $=2.93,95 \% \mathrm{Cl}=1.59-5.38)$, depression $(\mathrm{OR}=2.91,95 \% \mathrm{Cl}=1.65-5.12)$, and $\mathrm{RLS}(\mathrm{OR}=4.78,95 \% \mathrm{Cl}=1.41-$ 16.18). In addition, short sleep duration ( $\mathrm{OR}=4.33,95 \% \mathrm{Cl}=2.53-7.40)$ was also an important independent factor associated with insomnia.

Table 2. Univariate and multivari logistic regression of correlates of insomnia in older adults 
Model 1.

Characteristic

Characteristic

OR
(95\% Cl)

Unadjusted p value Adjusted OR

$0.99(0.97-$

1.02)

Age (years)

1.99 (1.29-

3.09)

$(95 \% \mathrm{Cl})$
Model 2.

Adjusted OR $\quad \mathrm{p}$

$\mathrm{p}$
value

$(95 \% \mathrm{Cl})$ p value

\begin{tabular}{|c|c|c|c|c|c|c|}
\hline Gender (female) & $\begin{array}{l}1.99(1.29- \\
3.09)\end{array}$ & $0.002^{\star}$ & $\begin{array}{l}2.25(1.33- \\
3.86)\end{array}$ & 0.003 & $\begin{array}{l}2.42(1.40- \\
4.19)\end{array}$ & 0.002 \\
\hline $\begin{array}{l}\text { Appetite within } 3 \text { months } \\
\text { (poor) }\end{array}$ & $\begin{array}{l}3.75(2.03- \\
6.93)\end{array}$ & $<0.001 *$ & $\begin{array}{l}3.02(1.53- \\
5.95)\end{array}$ & 0.001 & $\begin{array}{l}3.59(1.74- \\
7.39)\end{array}$ & 0.001 \\
\hline Exercise (none / irregular) & $\begin{array}{l}1.73(1.11- \\
2.68)\end{array}$ & $0.015^{\star}$ & - & - & - & - \\
\hline Sleeping pills usage (yes) & $\begin{array}{l}2.51(1.62- \\
3.89)\end{array}$ & $<0.001 *$ & $\begin{array}{l}1.96(1.21- \\
3.19)\end{array}$ & 0.006 & $\begin{array}{l}2.04(1.22- \\
3.40)\end{array}$ & 0.006 \\
\hline OSA risk (Medium-High) & $\begin{array}{l}2.00(1.24- \\
3.24)\end{array}$ & $0.005^{\star}$ & $\begin{array}{l}2.99(1.67- \\
5.33)\end{array}$ & $<0.001$ & $\begin{array}{l}2.93(1.59- \\
5.38)\end{array}$ & 0.001 \\
\hline Depression (GDS >=2) & $\begin{array}{l}4.10(2.53- \\
6.65)\end{array}$ & $<0.001 *$ & $\begin{array}{l}2.69(1.58- \\
4.56)\end{array}$ & $<0.001$ & $\begin{array}{l}2.91(1.65- \\
5.12)\end{array}$ & $<0.001$ \\
\hline RLS (yes) & $\begin{array}{l}2.49(1.31- \\
4.72)\end{array}$ & $0.005^{\star}$ & $\begin{array}{l}5.23(1.66- \\
16.40)\end{array}$ & 0.005 & $\begin{array}{l}4.78(1.41- \\
16.18)\end{array}$ & 0.012 \\
\hline Sleep duration ( $<=6 \mathrm{hrs}$ ) & $\begin{array}{l}3.47(2.19- \\
5.52)\end{array}$ & $<0.001^{*}$ & - & - & $\begin{array}{l}4.33(2.53- \\
7.40)\end{array}$ & $<0.001$ \\
\hline
\end{tabular}

* Logistic regression analysis with the forward stepwise method in model 1 was adjusted for all factors in the table (age, gender, poor appetite within 3 months, exercise, sleeping pills usage, risk of OSA, depression and RLS) except for sleep duration.

\# Results in model 2 were derived from Model 1 with sleep duration added as another covariate.

Abbreviations: OSA - obstructive sleep apnea, GDS- Geriatric Depression Scale, RLS - restless leg syndrome.

\section{The influence of gender on factors associated with insomnia}

Variables that showed significance in the entire sample remained significant in univariable analysis of the male and female groups evaluated separately (Table 3). After analyzing by multivariable logistic regression, only short sleep duration, medium-high risk of OSA, and depressive symptoms had the same association with insomnia in both genders. The association of appetite disappeared in the male group, while three factors (sleeping pills usage, non- or irregular exercise, RLS) became non-significant in the female group. Non- or irregular exercise, sleeping pills usage, and RLS had an independent association with insomnia only in men, while poor appetite within 3 months was associated with insomnia in women only.

Table 3. Gender differences in correlates of insomnia in older adults 


\begin{tabular}{|c|c|c|c|c|c|c|c|c|}
\hline \multirow{3}{*}{ Characteristic } & \multicolumn{4}{|c|}{ Male $(n=201)$} & \multicolumn{4}{|c|}{ Female $(n=199)$} \\
\hline & $\begin{array}{l}\text { Unadjusted } \\
\text { OR }\end{array}$ & p value & $\begin{array}{l}\text { Adjusted } \\
\text { OR }\end{array}$ & $\begin{array}{l}\mathrm{p} \\
\text { value }\end{array}$ & $\begin{array}{l}\text { Unadjusted } \\
\text { OR }\end{array}$ & p value & $\begin{array}{l}\text { Adjusted } \\
\text { OR }\end{array}$ & $\begin{array}{l}\mathrm{p} \\
\text { value }\end{array}$ \\
\hline & $(95 \% \mathrm{Cl})$ & & $(95 \% \mathrm{Cl})$ & & $(95 \% \mathrm{Cl})$ & & $(95 \% \mathrm{Cl})$ & \\
\hline Age (years) & $\begin{array}{l}0.99(0.96- \\
1.03)\end{array}$ & 0.594 & - & - & $\begin{array}{l}1.00(0.96- \\
1.04)\end{array}$ & 0.921 & - & - \\
\hline $\begin{array}{l}\text { Sleep duration } \\
(<=6 \mathrm{hrs})\end{array}$ & $\begin{array}{l}4.37(2.03- \\
9.43)\end{array}$ & $<0.001 *$ & $\begin{array}{l}4.52(1.94- \\
10.53)\end{array}$ & $<0.001$ & $\begin{array}{l}3.34(1.82- \\
6.12)\end{array}$ & $<0.001 *$ & $\begin{array}{l}4.23 \\
(2.11- \\
8.50)\end{array}$ & $<0.001$ \\
\hline $\begin{array}{l}\text { Appetite within } \\
3 \text { months } \\
\text { (poor) }\end{array}$ & $\begin{array}{l}3.87(1.36- \\
10.98)\end{array}$ & $0.011^{\star}$ & - & - & $\begin{array}{l}3.19(1.48- \\
6.89)\end{array}$ & $0.003^{*}$ & $\begin{array}{l}3.77 \\
(1.57- \\
9.08)\end{array}$ & 0.003 \\
\hline $\begin{array}{l}\text { Exercise } \\
\text { (none/irregular) }\end{array}$ & $\begin{array}{l}2.73(1.38- \\
5.40)\end{array}$ & $0.004^{\star}$ & $\begin{array}{l}2.61(1.18- \\
5.75)\end{array}$ & 0.018 & $\begin{array}{l}1.13(0.63- \\
2.04)\end{array}$ & 0.681 & - & - \\
\hline $\begin{array}{l}\text { Sleeping pills } \\
\text { usage (yes) }\end{array}$ & $\begin{array}{l}2.65(1.35- \\
5.20)\end{array}$ & $0.004^{\star}$ & $\begin{array}{l}2.67(1.22- \\
5.85)\end{array}$ & 0.014 & $\begin{array}{l}2.20(1.22- \\
3.95)\end{array}$ & $0.008^{*}$ & - & - \\
\hline $\begin{array}{l}\text { OSA risk } \\
\text { (Medium-High) }\end{array}$ & $\begin{array}{l}3.58(1.04- \\
12.32)\end{array}$ & $0.043^{*}$ & $\begin{array}{l}4.34(1.04- \\
18.07)\end{array}$ & 0.044 & $\begin{array}{l}3.06(1.68- \\
5.58)\end{array}$ & $<0.001^{\star}$ & $\begin{array}{l}2.56 \\
(1.31- \\
5.00)\end{array}$ & 0.006 \\
\hline $\begin{array}{l}\text { Depression } \\
(G D S>=2)\end{array}$ & $\begin{array}{l}4.46(2.06- \\
9.68)\end{array}$ & $<0.001 *$ & $\begin{array}{l}2.73(1.11- \\
6.75)\end{array}$ & 0.029 & $\begin{array}{l}3.42(1.82- \\
6.41)\end{array}$ & $<0.001^{\star}$ & $\begin{array}{l}3.48 \\
(1.70- \\
7.13)\end{array}$ & 0.001 \\
\hline RLS (yes) & $\begin{array}{l}7.29(1.29- \\
41.15)\end{array}$ & $0.025^{\star}$ & $\begin{array}{l}\text { 11.18(1.33- } \\
93.96)\end{array}$ & 0.026 & $\begin{array}{l}4.25(1.06- \\
16.97)\end{array}$ & $0.041^{*}$ & - & - \\
\hline
\end{tabular}

* Logistic regression analysis with the forward stepwise method stratified by gender was adjusted for age, sleep duration, poor appetite in 3 months, exercise, sleeping pills usage, OSA risk, depression and RLS.

Abbreviations: OSA - obstructive sleep apnea, GDS- Geriatric Depression Scale, RLS - restless leg syndrome.

\section{Discussion}

This cross-sectional study targeted a total of 400 older adult participants in outpatient clinics to comprehensively investigate the factors associated with insomnia, especially considering the influence of common sleep-related problems such as sleep duration, sleep medication usage, OSA and RLS, and intending to find the influence of gender on the associated factors. Results of the study showed that gender, poor appetite, not exercising regularly, depressive symptoms, and sleep-related conditions such as short sleep duration, sleeping pills usage, medium-high risk of OSA and RLS were factors associated with insomnia. Gender was also found to influence partially associated factors and insomnia. Exercise, sleeping pills usage, and RLS were relevant to insomnia in males alone, whereas poor appetite was relevant to insomnia in females only. Otherwise, significant associations were found between short sleep duration, medium-high risk of OSA and depressive symptoms and insomnia across genders.

The prevalence of insomnia depends on the method of identification. In the present study, the prevalence of AISdefined insomnia was $30 \%$ for all subjects and is similar to the reports in previous studies, which ranged from $30 \%$ to $48 \%$ [1]. After considering the influence of covariates, short sleep duration, sleeping pills usage, medium-high risk of OSA and RLS were also found to have independent associations with insomnia. Although the information of sleep 
duration was collected by questionnaires, the significant results were similar to the results derived from the general population using objective measures such as polysomnographic studies [32]. OSA and RLS are two common primary sleep disorders in older adults [33], and are frequently comorbid with insomnia, because all are associated with agingrelated physiologic and sleep architecture changes [12,34]. In the present study, $65.5 \%$ of participants demonstrated moderate-high risk of OSA and $4 \%$ of participants presented with the essential diagnostic features of RLS by IRLSSG. Both disorders are significantly associated with insomnia. Older adults tend to take sleeping pills to relieve sleep complaints, however, our report disclosed that those who use sleeping pills still tend to complain of insomnia symptoms. Few studies have focused on older adults who use hypnotic agents, except for those with insomnia. Although hypnotic agents improve sleep duration and latency, the magnitude of effect from sleep medications is small and potentially increases various adverse effects $[35,36]$. In addition, a variety of sleep medications have different mechanisms of action and different clinical effects on insomnia, which may influence patients' subjective feelings regarding sleep.

The present study is the first to identify the significant relationship between poor appetite and insomnia. Some clinicians have attributed poor appetite to be a manifestation of depression, but in the present study, the effect still existed in multivariable logistic regression analysis after adjusting for depressive symptoms identified by GDS-5. In addition, poor appetite may result in poor nutritional status, and previous studies have shown that nutritional inadequacy and malnutrition status identified by Mini Nutritional Assessment (MNA) scores correlate highly with insomnia [37, 38]. Furthermore, poor appetite may reduce the consumption of foods rich in melatonin, a hormone secreted by pituitary gland that is part of the sleep-wake cycle and is reported to improve sleep efficiency [39].

Although some epidemiologic studies reported a negative association between habitual exercise and insomnia, after adjusting only for demographic data and mood symptoms [40,41], the present study did not find similar significant associations after evaluating the effects of sleep-related problems. In addition, based on results of studies using quantitative exercise measurement instead of subjective self-reported questions as in the present study, both physical activity of moderate intensity and exercise training programs improved sleep symptoms and quality obviously [42, 43]. The effects of depression on insomnia remained strong across genders in the present study. A review of prospective studies has also recognized depression as a risk factor for sleep disturbances [10]. Various mechanisms have been proposed to help explain the findings relative to the effects of depression on sleep, including inflammatory regulations, genetic and familial factors, and the effects of social and environmental factors [44].

Similar to most previous studies, results of the present study show a clear relationship between female gender and insomnia $[9,10,14-17]$. However, gender not only influences the prevalence of insomnia, but also significantly affects the factors associated with insomnia in older adults. Few studies have investigated the differences in factors associated with insomnia between genders, especially not in older adults. A study of insomnia in Chinese adults aged 18-65 in Hong Kong revealed that socio-demographic factors (education level, occupation and retirement, marital status and environment) associated with insomnia were significantly different between genders, and though some common risk factors are found in both males and females, other risk factors were gender-specific [18]. Another study of the general population in Korea, however, found that the factors of education and occupation status were not significantly associated with insomnia [19]. In addition to the insomnia-associated factors mentioned above, and focusing on the older adult population, exercise and sleeping pills usage were shown to be relevant to insomnia in males alone, whereas appetite was associated with insomnia in females only. Although gender differences in insomnia represent a complex interaction between biological, psychological and social factors, the need remains to explain gender differences in factors associated with insomnia in future studies [45].

\section{Study contributions, implications, limitations}

Page $10 / 15$ 
The present study evaluated a group of older adult outpatients with a high prevalence of insomnia, and identified factors associated with insomnia, summarizing some potentially modifiable factors for further intervention. Evaluation with validated questionnaires provided conceivable results that could be followed and compared with other populations. Substantial differences found between genders in factors associated with insomnia added new perspectives in clinical practice.

Nevertheless, the study has several limitations. First, the cross-sectional design limits drawing causative conclusions, and sleep patterns were not evaluated by objective measures such as polysomnography or actigraphy. Second, collection of socio-demographic variables based on self-reported data or simple dichotomous yes/no questions may result in recall bias, especially among this older adult population, and there may still be some potential confounding factors left behind. Since the diagnosis of RLS was based on the four screening criteria of the IRLSSG, some mimicking diagnoses may be inevitably included. Third, subjects were recruited from outpatients in a single medical center and thus results may not be extrapolated to different settings or areas.

\section{Conclusions And Future Study}

The prevalence of insomnia in older adults is high, affecting about one in three older outpatients. Female gender, appetite, depression, and some sleep-related conditions (short sleep duration, OSA, RLS, and sleeping pills use) are significantly associated with insomnia. Significant differences are found between genders in factors associated with insomnia. Understanding gender differences identified in this study may help clinicians to modify associated factors when managing older adults with insomnia. Because gender differences in insomnia are the result of complex interactions between biological, psychological, genetic and social/lifestyle factors, further study is warranted to help explain gender differences among factors associated with insomnia.

\section{Abbreviations}

AIS: Athens Insomnia Scale, BMI: Body Mass Index, GDS-5: Geriatric Depression Scale-5, IRLSSG: International Restless Legs Syndrome Study Group, MNA: Mini Nutritional Assessment, OSA: obstructive sleep apnea, RLS: restless leg syndrome

\section{Declarations}

\section{Ethics approval and consent to participate}

The protocol for this study was approved by the Institutional Review Board of Kaohsiung Veterans General Hospital (VGHKS18-CT6-15). All study participants provided signed informed consent before any study-related procedure ensued.

\section{Consent for publication}

Not applicable.

\section{Availability of data and materials}

The datasets analysed during the current study are available from the corresponding author Chih-Kuang Liang on reasonable request. Please contact (ck.vghks@gmail.com) for data access.

\section{Competing interests}


All authors, Yu-Ting Peng, Ying-Hsin Hsu, Ming-Yueh Chou, Che-Sheng Chu, Chen-San Su, Chih-Kuang Liang, Yu-Chun Wang, Tsan Yang, Liang-Kung Chen, Yu-Te Lin have no conflicts of interest to declare, including financial and personal conflicts.

\section{Funding}

This study was sponsored by the Kaohsiung Veterans General Hospital [Grant number VGHKS18-CT6-15]. The funding organizations had no role in the design, methods, subject recruitment, data collection, analysis, or preparation of the article.

\section{Authors' contribution}

YTP drafted the manuscript, conducted the formal analysis, and interpreted the data.

YHH collected the data, drafted the manuscript, conducted the formal analysis, and interpreted the data.

MYC collected the data, interpreted the data and critically revised the manuscript for important intellectual content.

CSC collected the data, conducted the formal analysis, and interpreted the data.

CSS defined the study background and aims, defined the question guide and collected the data.

CKL conceptualized the paper, defined the study background and aims, defined the question guide, collected the data, interpreted the data and editing of the manuscript.

YCW, MD collected the data.

TY defined the study background and aims, defined the question guide, collected the data and contributed to interpretation of data.

LKC contributed to interpretation of data and production of the manuscript.

YTL contributed to interpretation of data and production of the manuscript.

\section{Acknowledgements}

We express our gratitude to the support from the Center for Geriatrics and Gerontology, Division of Neurology and Department of Psychiatry of Kaohsiung Veterans General Hospital.

\section{Author details}

${ }^{1}$ Center for Geriatrics and Gerontology, Kaohsiung Veterans General Hospital, Kaohsiung, Taiwan.

2 Department of Family Medicine, Kaohsiung Veterans General Hospital, Kaohsiung, Taiwan.

${ }^{3}$ Division of Neurology, Department of Internal Medicine, Kaohsiung Veterans General Hospital, Kaohsiung, Taiwan.

${ }^{4}$ Meiho University, Pingtung, Taiwan.

${ }^{5}$ Aging and Health Research Center, National Yang Ming Chiao Tung University, Taipei, Taiwan.

${ }^{6}$ Department of Geriatric Medicine, National Yang Ming Chiao Tung University School of Medicine, Taipei, Taiwan 
${ }^{7}$ Department of Psychiatry, Kaohsiung Veterans General Hospital, Kaohsiung, Taiwan.

${ }^{8}$ Department of Health Business Administration, Meiho University, Pingtung, Taiwan.

${ }^{9}$ Center for Geriatrics and Gerontology, Taipei Veterans General Hospital, Taipei, Taiwan.

10 Taipei Municipal Gan-Dau Hospital, Taipei, Taiwan

11 Department of Pharmacy, Tajen University, Pingtung, Taiwan

\section{References}

1. Patel D, Steinberg J, Patel P: Insomnia in the Elderly: A Review. J Clin Sleep Med 2018, 14(6):1017-1024.

2. Laugsand LE, Vatten LJ, Platou C, Janszky I: Insomnia and the risk of acute myocardial infarction: a population study. Circulation 2011, 124(19):2073-2081.

3. Yaffe K, Falvey CM, Hoang T: Connections between sleep and cognition in older adults. Lancet Neuro/2014, 13(10):1017-1028.

4. Fang H, Tu S, Sheng J, Shao A: Depression in sleep disturbance: A review on a bidirectional relationship, mechanisms and treatment. J Cell Mol Med 2019, 23(4):2324-2332.

5. Kyle SD, Morgan K, Espie CA: Insomnia and health-related quality of life. Sleep Med Rev 2010, 14(1):69-82.

6. Ohayon MM: Epidemiology of insomnia: what we know and what we still need to learn. Sleep Med Rev 2002, 6(2):97-111.

7. Zeng LN, Zong QQ, Yang Y, Zhang L, Xiang YF, Ng CH, Chen LG, Xiang YT: Gender Difference in the Prevalence of Insomnia: A Meta-Analysis of Observational Studies. Front Psychiatry 2020, 11:577429.

8. Patel NP, Grandner MA, Xie D, Branas CC, Gooneratne N: "Sleep disparity" in the population: poor sleep quality is strongly associated with poverty and ethnicity. BMC Public Health 2010, 10:475.

9. Tsou M-T: Prevalence and risk factors for insomnia in community-dwelling elderly in northern Taiwan. Journal of Clinical Gerontology and Geriatrics 2013, 4(3):75-79.

10. Smagula SF, Stone KL, Fabio A, Cauley JA: Risk factors for sleep disturbances in older adults: Evidence from prospective studies. Sleep Med Rev 2016, 25:21-30.

11. Aernout E, Benradia I, Hazo JB, Sy A, Askevis-Leherpeux F, Sebbane D, Roelandt JL: International study of the prevalence and factors associated with insomnia in the general population. Sleep Med 2021, 82:186-192.

12. Barthlen GM: Sleep disorders. Obstructive sleep apnea syndrome, restless legs syndrome, and insomnia in geriatric patients. Geriatrics 2002, 57(11):34-39, quiz 40.

13. Sweetman A, Melaku YA, Lack L, Reynolds A, Gill TK, Adams R, Appleton S: Prevalence and associations of comorbid insomnia and sleep apnoea in an Australian population-based sample. Sleep Med 2021, 82:9-17.

14. Wu CY, Su TP, Fang CL, Yeh Chang M: Sleep quality among community-dwelling elderly people and its demographic, mental, and physical correlates. J Chin Med Assoc 2012, 75(2):75-80.

15. Gambhir IS, Chakrabarti SS, Sharma AR, Saran DP: Insomnia in the elderly-A hospital-based study from North India. Journal of Clinical Gerontology and Geriatrics 2014, 5(4):117-121.

16. Uchmanowicz I, Markiewicz K, Uchmanowicz B, Koltuniuk A, Rosinczuk J: The relationship between sleep disturbances and quality of life in elderly patients with hypertension. Clin Interv Aging 2019, 14:155-165. 
17. Wang P, Song L, Wang K, Han X, Cong L, Wang Y, Zhang L, Yan Z, Tang S, Du Y: Prevalence and associated factors of poor sleep quality among Chinese older adults living in a rural area: a population-based study. Aging Clin Exp Res 2020, 32(1):125-131.

18. Li RHY, Wing YK, Ho SC, Fong SYY: Gender differences in insomnia-a study in the Hong Kong Chinese population. Journal of Psychosomatic Research 2002, 53:601-609.

19. La YK, Choi YH, Chu MK, Nam JM, Choi YC, Kim WJ: Gender differences influence over insomnia in Korean population: A cross-sectional study. PLoS One 2020, 15(1):e0227190.

20. Hwang LC, Bai Ch Fau - Chen C-J, Chen CJ: Prevalence of obesity and metabolic syndrome in Taiwan. J Formos Med Assoc 2006, 105(8):626-635.

21. Wen CP, David Cheng TY, Tsai SP, Chan HT, Hsu HL, Hsu CC, Eriksen MP: Are Asians at greater mortality risks for being overweight than Caucasians? Redefining obesity for Asians. Public Health Nutr 2009, 12(4):497-506.

22. Razak F, Anand SS, Shannon H, Vuksan V, Davis B, Jacobs R, Teo KK, McQueen M, Yusuf S: Defining obesity cut points in a multiethnic population. Circulation 2007, 115(16):2111-2118.

23. Rinaldi P, Mecocci P, Benedetti C, Ercolani S, Bregnocchi M, Menculini G, Catani M, Senin U, Cherubini A: Validation of the five-item geriatric depression scale in elderly subjects in three different settings. J Am Geriatr Soc 2003, 51(5):694-698.

24. Chin W-C, Liu C-Y, Lee C-P, Chu C-L: Validation of Five Short Versions of the Geriatric Depression Scale in the Elder Population in Taiwan. Taiwanese Journal of Psychiatry 2014, 28(3):156-163.

25. Arik G, Varan HD, Yavuz BB, Karabulut E, Kara O, Kilic MK, Kizilarslanoglu MC, Sumer F, Kuyumcu ME, Yesil Y et al: Validation of Katz index of independence in activities of daily living in Turkish older adults. Arch Gerontol Geriatr 2015, 61(3):344-350.

26. Soldatos CR, Dikeos DG, Paparrigopoulos TJ: The diagnostic validity of the Athens Insomnia Scale. J Psychosom Res 2003, 55(3):263-267.

27. Chiang H-L, Chen H-C, Bai C-H, Che H-H, Lee M-B, Lai S-H, Chou P: A Validation Study of the Chinese Version of the Athens Insomnia Scale. 2009, 23(1):43-52.

28. Hoevenaar-Blom MP, Spijkerman AM, Kromhout D, van den Berg JF, Verschuren WM: Sleep duration and sleep quality in relation to 12-year cardiovascular disease incidence: the MORGEN study. Sleep 2011, 34(11):1487-1492.

29. Chung F, Abdullah HR, Liao P: STOP-Bang Questionnaire: A Practical Approach to Screen for Obstructive Sleep Apnea. Chest 2016, 149(3):631-638.

30. Nagappa M, Liao P, Wong J, Auckley D, Ramachandran SK, Memtsoudis S, Mokhlesi B, Chung F: Validation of the STOP-Bang Questionnaire as a Screening Tool for Obstructive Sleep Apnea among Different Populations: A Systematic Review and Meta-Analysis. PLoS One 2015, 10(12):e0143697.

31. Allen RP, Picchietti D, Hening WA, Trenkwalder C, Walters AS, Montplaisi J: Restless legs syndrome: diagnostic criteria, special considerations, and epidemiology. Sleep Medicine 2003, 4(2):101-119.

32. Baglioni C, Regen W, Teghen A, Spiegelhalder K, Feige B, Nissen C, Riemann D: Sleep changes in the disorder of insomnia: a meta-analysis of polysomnographic studies. Sleep Med Rev 2014, 18(3):195-213.

33. Neikrug AB, Ancoli-Israel S: Sleep disorders in the older adult - a mini-review. Gerontology 2010, 56(2):181-189.

34. Gooneratne NS, Vitiello MV: Sleep in older adults: normative changes, sleep disorders, and treatment options. Clin Geriatr Med 2014, 30(3):591-627.

35. Holbrook AM, Crowther R, Lotter A, Cheng C, King D: Meta-analysis of benzodiazepine use in the treatment of insomnia. CMAJ 2000, 162(2):225-233. 
36. Glass J, Lanctot KL, Herrmann N, Sproule BA, Busto UE: Sedative hypnotics in older people with insomnia: metaanalysis of risks and benefits. BMJ 2005, 331(7526):1169.

37. Matsuura N, Saito A, Takahashi O, Rahman M, Tajima R, Mabashi-Asazuma H, lida K: Associations between nutritional adequacy and insomnia symptoms in Japanese men and women aged 18-69 years: a cross-sectional study. Sleep Health 2020, 6(2):197-204.

38. Soysal P, Smith L, Dokuzlar O, Isik AT: Relationship Between Nutritional Status and Insomnia Severity in Older Adults. J Am Med Dir Assoc 2019, 20(12):1593-1598.

39. Meng X, Li Y, Li S, Zhou Y, Gan RY, Xu DP, Li HB: Dietary Sources and Bioactivities of Melatonin. Nutrients 2017, $9(4)$.

40. Inoue S, Yorifuji T Fau - Sugiyama M, Sugiyama M Fau - Ohta T, Ohta T Fau - Ishikawa-Takata K, Ishikawa-Takata K Fau - Doi H, Doi H: Does habitual physical activity prevent insomnia? A cross-sectional and longitudinal study of elderly Japanese. J Aging Phys Act 2013, 21(2):119-139.

41. Kim K, Uchiyama M, Okawa M, Liu X, Ogihara R: An epidemiological study of insomnia among the Japanese general population. Sleep 2000, 23(1):41-47.

42. Yang $\mathrm{P}-\mathrm{Y}, \mathrm{Ho} \mathrm{K}-\mathrm{H}$, Chen $\mathrm{H}-\mathrm{C}$, Chien $\mathrm{M}-\mathrm{Y}$ : Exercise training improves sleep quality in middle-aged and older adults with sleep problems: a systematic review. Journal of Physiotherapy 2012, 58(3):157-163.

43. Vanderlinden J, Boen F, van Uffelen JGZ: Effects of physical activity programs on sleep outcomes in older adults: a systematic review. International Journal of Behavioral Nutrition and Physical Activity 2020, 17(1).

44. Alvaro PK, Roberts RM, Harris JK: A Systematic Review Assessing Bidirectionality between Sleep Disturbances, Anxiety, and Depression. Sleep 2013, 36(7):1059-1068.

45. Suh S, Cho N, Zhang J: Sex Differences in Insomnia: from Epidemiology and Etiology to Intervention. Curr Psychiatry Rep 2018, 20(9):69. 